\title{
Perfil clínico-laboratorial de pacientes hospitalizados acometidos por lesão por pressão
}

\section{Clinical and laboratory profile of hospitalized patients affected by pressure injury Perfil clinico-laboratorio de pacientes hospitalizados afectados por lesion por presion}

\author{
Marcela Gama Santana Moreira* ${ }^{*}$ Silvia de Magalhães Simões², Caíque Jordan Nunes Ribeiro³
}

ORCID IDs

Moreira MGS (D) https://orcid.org/0000-0002-4951-2504

Simões SM (D) https://orcid.org/0000-0003-2751-7993

Ribeiro CJN (D) https://orcid.org/0000-0001-9767-3938

\section{COMO CITAR}

Moreira MGS; Simões SM; Ribeiro CJN. Perfil clínico-laboratorial de pacientes hospitalizados acometidos por lesão por pressão. ESTIMA, Braz. J. Enterostomal Ther., 2020, 18: e2220. https://doi. org/10.30886/estima.v18.885_PT

\section{RESUMO}

Objetivo: Caracterizar o perfil clínico-laboratorial de pacientes hospitalizados acometidos por lesão por pressão (LP). Método: Estudo retrospectivo e descritivo, que incluiu dados de prontuários eletrônicos de 95 pacientes acometidos por LP durante a hospitalização. Resultados: Houve predomínio do sexo feminino (52,6\%), média de idade 74,8 \pm 14 anos, tempo médio de internação foi de $76,9 \pm 88,8$ dias. A maioria esteve internada na unidade de terapia intensiva, com uma média de 17,86 $\pm 36,58$ dias. Com relação à condição clínica, 60\% estavam em uso de ventilador mecânico quando desenvolveram a LP, 37,9\% tinham a necessidade de hemodiálise, 30,4\% foram diagnosticados com algum grau de desnutrição energético-proteica e 54,7\% evoluíram para o óbito. As comorbidades mais frequentes foram hipertensão $(63,16 \%)$, diabetes $(43,16 \%)$ e neuropatia $(33,68 \%)$. Quanto ao perfil laboratorial, hipoalbuminemia (97,3\%), hiperglicemia (87,8\%), anemia (84,4\%) e hiperuremia (78,9\%) estiveram presentes em mais de dois terços da amostra. Conclusão: Este estudo permitiu conhecer o perfil de pacientes acometidos por LP durante internação hospitalar, o que pode servir de base para desenvolver ações preventivas eficazes embasadas cientificamente.

DESCRITORES: Adulto; Estomaterapia; Hospitalização; Lesão por pressão; Pele.

\footnotetext{
1. Universidade Federal de Sergipe - Programa de Pós-graduação em Ciências da Saúde - Aracaju (SE), Brasil.

2. Universidade Federal de Sergipe - Centro de Ciências Biológicas e da Saúde - Departamento de Medicina - Aracaju (SE), Brasil.

3. Instituto Federal de Educação, Ciência e Tecnologia de Sergipe - Coordenadoria de Saúde Escolar - São Cristóvão (SE), Brasil.

*Autora correspondente: marcelinha_gama@hotmail.com

Recebido: Mai. 07, 2020 | Aceito: Set. 23, 2020
} 


\begin{abstract}
Objective: To characterize the clinical and laboratory profile of hospitalized patients affected by pressure injury (PI). Method: Retrospective and descriptive study, which included data from electronic medical records of 95 patients affected by PI during hospitalization. Results: There was a predominance of females (52.6\%), mean age $74.8 \pm 14$ years, mean hospital stay was $76.9 \pm 88.8$ days. Most were admitted to the intensive care unit, with an average of $17.86 \pm 36.58$ days. Regarding the clinical condition, $60 \%$ were using a mechanical ventilator when they developed PI, 37.9\% needed hemodialysis, 30.4\% were diagnosed with some degree of protein-energy malnutrition and 54.7\% progressed to death. The most frequent comorbidities were hypertension (63.16\%), diabetes (43.16\%) and neuropathy (33.68\%). As for the laboratory profile, hypoalbuminemia (97.3\%), hyperglycemia (87.8\%), anemia (84.4\%) and hyperuremia (78.9\%) were present in more than two thirds of the sample.Conclusion: This study allowed to know the profile of patients affected by PI during hospitalization, which can serve as a basis for developing scientifically based effective preventive actions.
\end{abstract}

DESCRIPTORS: Adult; Stomatherapy; Hospitalization; Pressure injury; Skin.

\title{
RESUMEN
}

Objetivo: caracterizar el perfil clínico y de laboratorio de pacientes hospitalizados afectados por lesiones por presión (LP). Método: estudio retrospectivo y descriptivo, que incluyó datos de historias clínicas electrónicas de 95 pacientes afectados por LP durante la hospitalización. Resultados: hubo predominio del sexo femenino (52,6\%), edad media 74,8 14 años, estancia hospitalaria media $76,9 \pm 88,8$ días. La mayoría ingresó en la unidad de cuidados intensivos, con una media de 17,86₫36,58 días. En cuanto a la situación clínica, el 60\% utilizaba ventilador mecánico cuando desarrollaron LP, el 37,9\% necesitó hemodiálisis, el 30,4\% fue diagnosticado con algún grado de desnutrición proteico-energética y el 54,7\% progresó a muerte. Las comorbilidades más frecuentes fueron hipertensión (63,16\%), diabetes (43,16\%) y neuropatía (33,68\%). En cuanto al perfil de laboratorio, la hipoalbuminemia (97,3\%), hiperglucemia (87,8\%), anemia $(84,4 \%)$ e hiperuremia $(78,9 \%)$ estuvieron presentes en más de dos tercios de la muestra. Conclusión: este estudio permitió conocer el perfil de los pacientes afectados por LP durante la hospitalización, lo que puede servir de base para desarrollar acciones preventivas efectivas con base científica.

DESCRIPTORES: Adulto; Hospitalización; Úlcera por presión; Piel.

\section{INTRODUÇÃO}

A lesão por pressão (LP) é definida como região da pele com sofrimento tecidual devido à intensa e/ou prolongada pressão nos tecidos moles, geralmente nas áreas de proeminências ósseas, com possibilidade de associação ao cisalhamento, ou ainda pode estar relacionada a dispositivos médicos ou outro tipo de artefato ${ }^{1}$. O dano tecidual decorre da pressão externa no tecido, causada pela superfície de contato ser superior à pressão de perfusão capilar por um tempo maior ao necessário para recuperação da isquemia gerada ${ }^{2}$

O grau de comprometimento tecidual é considerado para estadiamento das LPs, a quais podem ser classificadas em: estágio 1 (pele íntegra com hiperemia não branqueável); estágio 2 (perda parcial da pele, com exposição da derme, ou bolha com conteúdo seroso); estágio 3 (perda total da pele, com exposição do tecido subcutâneo, além de poder apresentar granulação, esfacelo e necrose); estágio 4 (perda total da pele, com exposição de outras estruturas); tissular profunda (pele íntegra ou não, com coloração vermelha escura, marrom ou púrpura que não embranquece, ou bolha com conteúdo sanguinolento); e não classificável (perda da pele em sua espessura total encoberta por esfacelo ou escara) ${ }^{1}$.

As LPs estágios 3, 4 e não classificável, quando adquiridas após admissão em instituições de saúde, são consideradas "never events", ou seja, eventos adversos inequívocos, sérios e geralmente evitáveis ${ }^{3}$. Estas lesões originadas por pressão constituem um problema mundial altamente prevalente, representando a condição mais grave da integridade da pele prejudicada por resultarem em inúmeros desfechos negativos, tais como complicações clínicas, impactos físicos e psicossociais que repercutem diretamente na qualidade de vida dos indivíduos ${ }^{4-6}$. Configura-se um indicador de qualidade da assistência à saúde ${ }^{7}$ e o seu surgimento pode levar à judicialização de instituições e profissionais de saúde ${ }^{8}$.

Essas complicações elevam o tempo de hospitalização, as taxas de readmissão hospitalar, as taxas de morbimortalidade, a necessidade de cuidados intensivos, a carga de trabalho para equipe de enfermagem, com consequente aumento do custo para o sistema de saúde ${ }^{9-10}$.

É importante destacar a importância da avaliação do risco de desenvolvimento de LP para prevenção e 
intervenção precoce. Para execução do gerenciamento de risco, é fundamental que os profissionais conheçam a população suscetível, a fim de intervir com ações direcionadas à minimização dos fatores de risco inerentes ou não ao paciente. Além disso, existem ferramentas que auxiliam na avaliação sistemática do risco para LP, como a escala de Braden, traduzida para várias línguas, sendo a mais utilizada mundialmente ${ }^{11}$. Por outro lado, o julgamento clínico é imprescindível, pois esta escala avalia apenas a percepção sensorial, umidade, mobilidade, atividade, nutrição, fricção e cisalhamento ${ }^{11}$, ou seja, outros fatores de risco que não são mensurados nessa escala podem estar diretamente associados ao aparecimento de LP.

Nesse contexto, diante da prática profissional voltada para o gerenciamento de LP em ambiente hospitalar, surgiu a motivação para realização desse estudo. Pontua-se a importância do conhecimento sobre o perfil clínicoepidemiológico dos pacientes que desenvolvem LP em ambiente hospitalar, uma vez que as características encontradas podem ser semelhantes em diferentes cenários. Destarte, a realização de estudos nessa área pode contribuir para melhora na tomada de decisão da equipe de enfermagem quanto às estratégias preventivas para LP. De tal modo, o êxito na prevenção destas lesões otimiza os resultados dos indicadores que refletem a qualidade dos serviços de enfermagem prestados, como também favorece a qualidade de vida dos indivíduos.

\section{OBJETIVO}

Caracterizar o perfil clínico-laboratorial de pacientes hospitalizados acometidos por LP.

\section{MÉTODOS}

Estudo descritivo retrospectivo a partir de dados secundários, coletados por meio de pesquisa documental. Este tipo de pesquisa foi realizado a partir de documentos retrospectivos, não fraudados, sem tratamento analítico ${ }^{12}$.

A pesquisa foi realizada em um hospital geral da rede privada na cidade de Aracaju no estado de Sergipe, Brasil. Trata-se de um hospital de alta complexidade, composto por instalações de urgência, hemodinâmica, centro cirúrgico, unidade de terapia intensiva (UTI) adulta e pediátrica e unidades de internação e que atende diversas especialidades médicas.

A instituição possui comissão de pele composta por oito enfermeiras capacitadas na prevenção e tratamento de lesões de pele e/ou com especialização em enfermagem em dermatologia ou estomaterapia, a fim de realizar o gerenciamento de LPs. Os pacientes assistidos por esta equipe são captados por triagem ou por solicitação dos demais profissionais de saúde (médicos, enfermeiros e técnicos de enfermagem). Esta comissão é acionada em situações em que pacientes tem possibilidade de desenvolver lesões de pele, ou em pacientes incontinentes em uso de fralda, portadores de estomia ou, ainda, quando a aplicação da escala de Braden, na avaliação de pacientes, resulta em escore $\leq 14$.

A equipe de enfermagem executa diariamente atividades determinadas nos protocolos institucionais de prevenção e tratamento de lesão, como, por exemplo: a inspeção da pele, aplicação da escala de Braden, realização de curativos, instalação de coberturas preventivas e cuidados ao paciente com estomia. A comissão de pele gerencia tais atividades por meio da avaliação do paciente e do prontuário e da confecção da prescrição diária de coberturas preventivas e curativos, além de notificar novos casos de LP dentro da instituição, conforme recomendações da National Pressure Injury Advisory Panel (NPIAP) ${ }^{1}$.

A amostra se deu por conveniência, composta pelos casos de pacientes maiores de 18 anos que desenvolveram LP no ambiente hospitalar no período de junho de 2017 a junho de 2018, totalizando 95 participantes. Os pacientes que desenvolveram apenas lesão por dispositivo médico foram excluídos, uma vez que, na análise dos casos feita pelos gestores, observou-se que a maioria foi por falha no cumprimento do protocolo de prevenção de LP. Os dados clínico-laboratoriais destes pacientes foram extraídos dos prontuários eletrônicos, disponíveis no sistema da instituição, na qual um dos autores tem acesso, entre os meses de setembro de 2018 a março de 2019.

Os dados foram organizados e tabulados em planilhas do Microsoft Office Excel $2013^{\circledR}$ e exportados para o programa $R$ Core Team 2019. As variáveis categóricas foram expressas em frequências absolutas e percentuais, e as quantitativas em médias e desvios-padrão. Foram utilizados os testes Quiquadrado de Pearson, com e sem simulações de MonteCarlo para verificar a associação entre variáveis categóricas e Mann-Whitney para amostras independentes para verificar a diferença das medianas entre os grupos. 
Esse estudo foi aprovado pelo Comitê de Ética em Pesquisa institucional (CAAE no 89872918.4.0000.5546) e respeitou as recomendações éticas da Resolução 466/2012 do Conselho Nacional de Saúde e da Declaração de Helsinque.

\section{RESULTADOS}

A amostra foi composta por 95 pacientes, dos quais 50 eram mulheres (52,6\%), com média de idade 74,8 \pm 14 anos, cujo tempo médio de internação foi de $76,9 \pm 88,8$ dias. Desses, 76 (80\%) estiveram na UTI, com uma média de 17,86 $\pm 36,58$ dias de internação. Quinze $(15,8 \%)$ estavam sob cuidados paliativos. O desfecho óbito foi verificado em 52 pacientes (54,7\%). Com relação à condição clínica, 57 pacientes (60\%) estavam em uso de ventilador mecânico quando desenvolveram a LP, 36 (37,9\%) tinham a necessidade de hemodiálise e 28 $(30,4 \%)$ foram diagnosticados com algum grau de desnutrição energético-proteica (DEP).

Apenas um (1,05\%) paciente acometido por LP não apresentava doença de base, $20(21,07 \%)$ apresentavam somente uma, $30(31,57 \%)$ pacientes duas e $44(46,31 \%)$ com três ou mais. As principais comorbidades encontradas em prontuário estão apontadas na Tabela 1.

Tabela 1. Comorbidades dos pacientes acometidos por lesão por pressão. Aracaju (SE), Brasil - setembro de 2018 a março de 2019.

\begin{tabular}{|c|c|}
\hline Comorbidades & n (\%) \\
\hline Hipertensão arterial & $60(63,16)$ \\
\hline Diabetes mellitus & $41(43,16)$ \\
\hline Neuropatia & $32(33,68)$ \\
\hline Cardiovasculopatia & $26(27,36)$ \\
\hline Doença oncológica & $26(27,36)$ \\
\hline Nefropatia & $20(21,05)$ \\
\hline Pneumopatia & $14(14,73)$ \\
\hline Hepatopatia & $11(11,57)$ \\
\hline
\end{tabular}

As terapias medicamentosas utilizadas pelos pacientes com LP estão apresentadas na Tabela 2. Os fármacos antibióticos foram os mais prescritos, enquanto os quimioterápicos os menos frequentemente utilizados.

Tabela 2. Medicações em uso pelos pacientes com lesão por pressão. Aracaju (SE), Brasil - setembro de 2018 a março de 2019.

\begin{tabular}{|c|c|}
\hline Variável & Valores n (\%) \\
\hline Drogas vasoativas & $54(56,8)$ \\
\hline Sedativos & $47(49,5)$ \\
\hline Corticosteroides & $65(68,4)$ \\
\hline Quimioterápicos & $6(6,3)$ \\
\hline Antibióticos & $82(86,3)$ \\
\hline
\end{tabular}

Quanto aos exames laboratoriais, quase 70\% dos pacientes apresentaram resultados alterados, com exceção da contagem de plaquetas e nível de creatinina, nos quais menos de
35\% dos indivíduos apresentaram alteração. A Tabela 3 apresenta a média dos valores dos exames e os resultados categorizados conforme os valores de referência do local 
da pesquisa. Entre os achados que mais chamam atenção estão a hipoalbuminemia $(97,3 \%)$, hiperglicemia $(87,8 \%)$, anemia $(84,4 \%)$ e hiperuremia $(78,9 \%)$ estiveram presentes em mais da metade da amostra.

Tabela 3. Perfil laboratorial de pacientes acometidos por lesão por pressão. Aracaju (SE), Brasil - setembro de 2018 a março de 2019.

\begin{tabular}{|c|c|c|c|}
\hline $\begin{array}{c}\text { Exame } \\
\text { (valor de referência) }\end{array}$ & Média \pm DP & Alteração & n (\%) \\
\hline \multirow{2}{*}{$\begin{array}{l}\text { Hemoglobina } \\
(12-16 \mathrm{~g} / \mathrm{dL})\end{array}$} & \multirow{2}{*}{$9,9 \pm 2,1$} & $\downarrow$ & $76(84,4)$ \\
\hline & & $\uparrow$ & $2(2,2)$ \\
\hline Leucócitos & \multirow{2}{*}{$15.560 \pm 9.685,1$} & $\downarrow$ & $4(4,4)$ \\
\hline$\left(4.000-11.000 / \mathrm{mm}^{3}\right)$ & & $\uparrow$ & $58(64,4)$ \\
\hline \multirow{2}{*}{$\begin{array}{c}\text { Plaquetas } \\
\left(130.000-400.000 / \mathrm{mm}^{3}\right)\end{array}$} & \multirow{2}{*}{$230.511,1 \pm 123.625,1$} & $\downarrow$ & $22(24,4)$ \\
\hline & & $\uparrow$ & $9(10)$ \\
\hline $\begin{array}{c}\text { Ureia } \\
(<50 \mathrm{mg} / \mathrm{dL})\end{array}$ & $89,5 \pm 50,8$ & $\uparrow$ & $71(78,9)$ \\
\hline $\begin{array}{c}\text { Creatinina } \\
(<1,25 \mathrm{mg} / \mathrm{dL})\end{array}$ & $1,4 \pm 1,3$ & $\uparrow$ & $29(32,2)$ \\
\hline $\begin{array}{c}\text { Albumina } \\
(3,5-5,0 \mathrm{~g} / \mathrm{dL})\end{array}$ & $2,6 \pm 0,4$ & $\downarrow$ & $71(97,3)$ \\
\hline $\begin{array}{c}\text { Glicemia } \\
(60-100 \mathrm{mg} / \mathrm{dL})\end{array}$ & $175,2 \pm 90,3$ & $\uparrow$ & $43(87,8)$ \\
\hline
\end{tabular}

$\mathrm{DP}=$ desvio padrão.

A média do escore da escala de Braden foi 10,5 $\pm 2,5$, classificado como alto risco, e a média do intervalo de tempo para desenvolvimento das LPs foi de 25,8 $\pm 43,09$ dias. A LP “estágio 3" demandou maior tempo de hospitalização para se desenvolver, 55,6 \pm 80,8 dias (Tabela 4). A LP "tissular profunda" foi a categoria mais notificada $(42,5 \%)$, seguido por “estágio 2"(28\%). Não foram notificadas lesões em “estágio 4".

A superfície corporal mais acometida pela LP foi a região glútea $(39,4 \%)$, seguida pela região sacrococcígea $(19,3 \%)$ e os calcâneos $(9,3 \%)$ (Fig. 1).

Tabela 4. Medicações em uso pelos pacientes com lesão por pressão. Aracaju (SE), Brasil - setembro de 2018 a março de 2019.

\begin{tabular}{|c|c|c|c|c|}
\hline \multirow{3}{*}{ Classificação } & \multirow{3}{*}{$\%$} & \multicolumn{2}{|c|}{ Tempo para lesão (dias) } & \multirow{3}{*}{$p$-valor } \\
\hline & & Presente & Ausente & \\
\hline & & \multicolumn{2}{|c|}{ Média \pm DP } & \\
\hline Não classificável & 1,5 & 10 & $26,2 \pm 43,5$ & $0,640^{w}$ \\
\hline Estágio 1 & 15,8 & $31,4 \pm 76,4$ & $24,5 \pm 31,3$ & $0,126^{w}$ \\
\hline Estágio 2 & 28 & $24,9 \pm 35,4$ & $26,5 \pm 47,9$ & $0,912^{w}$ \\
\hline Estágio 3 & 12,3 & $55,6 \pm 80,8$ & $19,8 \pm 27,6$ & $0,003^{w}$ \\
\hline Tissular profunda & 42,5 & $18,7 \pm 19,3$ & $30,8 \pm 53,4$ & $0,650^{w}$ \\
\hline
\end{tabular}

$\mathrm{DP}=$ desvio-padrão; w Teste de Mann-Whitney. 


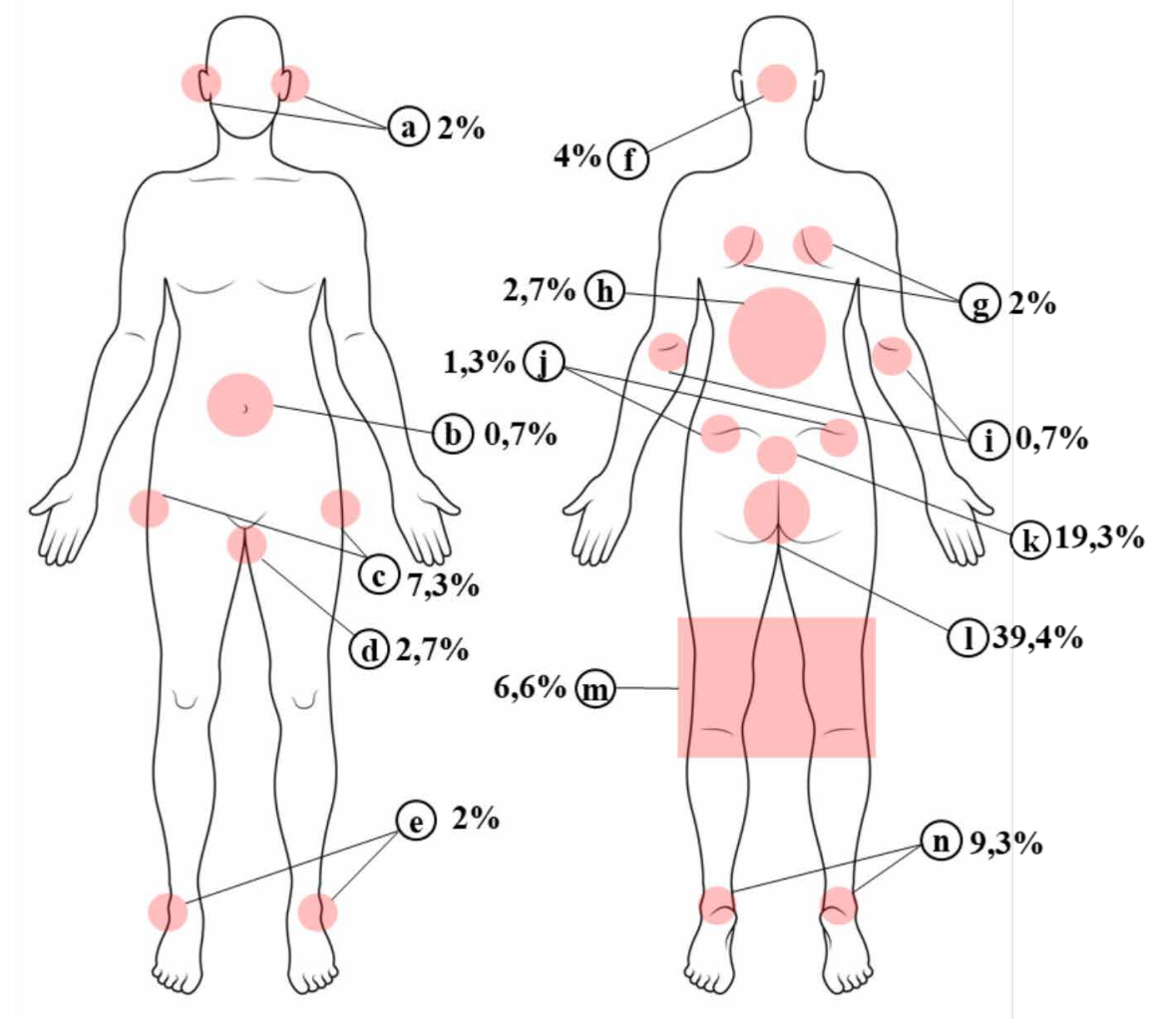

LEGENDA

(a) Pavilhão auricular

(b) Abdome

(c) Trocanter

(d) Ísquio

(e) Maléolo lateral

(f) Cabeça

(g) Escápula

(h) Dorso

(i) Cotovelo

(j) Crista ilíaca

(k) Sacrococcígea

(1) Glúteo

(m) Membros inferiores

(n) Calcâneo

Figura 1. Regiões acometidas pela lesão por pressão. Aracaju (SE), Brasil - setembro de 2018 a março de 2019.

\section{DISCUSSÃO}

Os resultados deste estudo consistem na caracterização do perfil clínico-laboratorial dos pacientes que desenvolveram LP durante hospitalização. Quanto à idade, observou-se a idade avançada dos indivíduos (> 70 anos). Sabe-se que o envelhecimento causa modificações estruturais e funcionais da pele. Algumas alterações presentes na pele do idoso que podem comprometer a tolerância tecidual à pressão são: espessura cutânea reduzida, junção entre derme e epiderme prejudicada, divisão celular limitada, gordura subcutânea diminuída, resposta vascular lentificada e inervação e sensibilidade alteradas ${ }^{13}$.

Além disso, a população idosa representa um grupo etário com estado funcional prejudicado, atividade e mobilidade reduzidas, como também maior tempo de hospitalizaçãa ${ }^{14}$. O estado funcional comprometido foi evidenciado na amostra, uma vez que a maioria tinha pelo menos um diagnóstico de doença de base. Resultados de um estudo apontaram para alta associação entre as doenças sistêmicas e a existência de $\mathrm{LP}^{15}$.
O óbito foi o desfecho da internação em mais da metade dos pacientes com LP. Pesquisadores avaliaram a taxa de sobrevida em pacientes hospitalizados e identificaram que a média do tempo de sobrevida do grupo de indivíduos portadores de LP foi consideravelmente menor do que o grupo sem LP (94 vs. 414 dias $)^{15}$. Embora estas lesões não sejam um fator causal de óbito durante internação hospitalar, elas elevam o risco de processos infecciosos e de desnutrição hospitalar9.

A média do escore pela escala de Braden revelou "alto risco" para desenvolvimento de LP. Esse achado corrobora o perfil dos pacientes analisados, uma vez que foi verificado um número considerável de pacientes com LP em uso de medicações sedativas, ventilação mecânica e com necessidade de hemodiálise. Salienta-se que pacientes em uso de sedação, analgesia ou relaxante geralmente não percebem pressão tecidual ou não conseguem reagir adequadamente 9 .

Destaca-se que a maioria dos participantes deste estudo desenvolveram LP na UTI. A indicação de UTI pode ser para controle de sinais vitais e, principalmente, para intervenções clínicas em pacientes hemodinamicamente instáveis e/ou 
com falência de um único ou múltiplos órgãos. Um estudo realizado em hospitais públicos na Austrália evidenciou que os pacientes alocados em UTI têm 3,8 vezes mais chances de desenvolver LP quando comparado a pacientes internados em outras unidades16. Isto posto, os pacientes internados em UTI devem ser considerados de alto risco para o surgimento de LP.

Adicionalmente, os medicamentos vasoativos utilizados no contexto da UTI têm a finalidade de manter a pressão arterial, otimizando o fluxo sanguíneo rico em oxigênio para os órgãos vitais e, consequentemente, diminuindo a perfusão para o tecido cutâneo e outros tecidos sob pressãa ${ }^{17}$.

Quanto aos exames laboratoriais, é notável a presença de anemia e hipoalbuminemia entre os portadores de LP. A anemia tem sido documentada em outros estudos como possível contribuinte para o surgimento de $\mathrm{LP}^{18-19}$ Ademais, influencia na resistência dos tecidos à pressão por conta da possibilidade de reduzir a oxigenação tecidual e, consequentemente, comprometer o processo cicatricial ${ }^{18}$ Também, pode ser uma condição resultante da influência combinada de doenças sistêmicas avançadas, como doença oncológica, disfunção renal crônica e infecções sistêmicas ${ }^{15}$.

Quanto à hipoalbuminemia presente na maioria $(97,3 \%)$ dos pacientes com LP deste estudo, outras pesquisas também demonstram resultados semelhantes em diferentes cenários de saúde ${ }^{15,20}$. A albumina é uma proteína vista como marcadora de nutrição, como também da pressão oncótica, que é reduzida quando os níveis de albumina se encontram baixos, ocasionando o edema e consequentemente comprometendo a tolerância tecidual ${ }^{20}$. Além disso, é importante destacar que a desnutrição ocasiona redução da massa muscular, disfunção imunológica, consequentemente comprometendo a resposta inflamatória, diminuição da camada subcutânea e afrouxamento da junção derme e epiderme ${ }^{17,21}$. Essas condições acentuam proeminências ósseas e/ou proporcionam maior risco de ruptura cutânea, sendo facilmente encontradas em pessoas imobilizadas e idosas 5 .

O estágio mais notificado foi "tissular profunda", seguido por "estágio 2", divergindo de outros estudos que apontam o "estágio 2" como o mais frequente ${ }^{9,21}$. Ambos os estágios são definidos como danos leves pela NPIAP, todavia, podem evoluir para estágios mais avançados, atingindo tecidos mais profundos, em situações em que não sejam realizadas ações adequadas de tratamento1. Destaca-se, ainda, as notificações de 17 lesões “estágio 3" e duas "não classificável", correspondendo ao never event ${ }^{3}$.
O estágio mais encontrado entre os pacientes que evoluíram a óbito foi tissular profunda. Ressalta-se que estas lesões podem piorar e atingir estruturas mais profundas, tornando-se extensas e cavitárias, quando as condições clínicas do paciente não forem favoráveis ao processo de cicatrização ${ }^{22}$.O tempo para desenvolvimento da LP “estágio 3 " foi consideravelmente superior, comparado ao dos outros estágios notificados. O tempo pode ser associado ao nível de comprometimento tecidual causado pela lesão, no qual o "estágio 3" atinge até o tecido subcutâneo ${ }^{1}$.

No tocante à topografia, a região corporal mais atingida foi a região glútea, seguida pela região sacrococcígea, divergindo de outras pesquisas que relatam a região sacra como a mais acometida $^{9,23}$. Apesar desse contraste, estas superfícies corporais são próximas, além de serem regiões de apoio quando o indivíduo assume o decúbito dorsal. Além disso, esses locais são frequentemente acometidos pela dermatite associada à incontinência (DAI) fecal ou urinária, sendo esta considerada um fator predisponente de $\mathrm{LP}^{24}$.

Diante do conhecimento do perfil dos indivíduos acometidos por LP no ambiente hospitalar, é possível traçar ações de promoção da saúde e prevenção para a população hospitalizada. $\mathrm{O}$ resultado desta pesquisa permitiu identificar outras variáveis, não contempladas na escala de Braden, como envolvidas no surgimento de LP. Assim, estes resultados reforçam a importância da avaliação individual do paciente, além da aplicação da escala de Braden. Ressalta-se que as medidas de prevenção para manutenção da integridade cutânea durante a internação hospitalar são extremamente necessárias para atingir bons desfechos quanto aos indicadores que mensuram a qualidade da assistência de enfermagem.

O estudo apresentou como limitação a realização da pesquisa em apenas um hospital. Contudo, os resultados encontrados nesta pesquisa sugerem a realização de estudos longitudinais e prospectivos que possam elucidar modelos estatísticos de predição de risco de LP que levem em consideração os possíveis fatores contribuintes descritos em nossos achados.

\section{CONCLUSÃO}

A caracterização do perfil clínico-laboratorial de pacientes hospitalizados acometidos por LP pode favorecer o planejamento de ações preventivas eficazes e cientificamente embasadas. Além disso, pode contribuir para a melhoria da assistência de enfermagem e obtenção de melhores 
resultados relacionados aos indicadores da qualidade da assistência hospitalar.

De tal forma, o conhecimento do perfil clínicolaboratorial dos indivíduos hospitalizados que desenvolvem LP favorece o trabalho da equipe de saúde, principalmente da enfermagem. Nos casos de grande fluxo de atendimentos e cuidado a pacientes críticos, tal conhecimento auxiliará o enfermeiro na priorização das atividades e na sistematização da assistência de enfermagem, promovendo segurança ao paciente e qualidade nas intervenções realizadas.

\section{CONTRIBUIÇÃO DOS AUTORES}

\author{
Conceitualização: Moreira MGS e Simões SM;
} Metodologia: Moreira MGS; Simões SM e Ribeiro CJN; Investigação: Moreira MGS; Redação - Primeira versão: Moreira MGS; Redação - Revisão \& Edição: Moreira MGS; Simões SM e Ribeiro CJN; Aquisição de Financiamento: Moreira MGS e Simões SM; Recursos: Moreira MGS; Simões SM e Ribeiro CJN; Supervisão: Simões SM.

\section{REFERÊNCIAS}

1. Edsberg LE, Black JM, Goldberg M, McNichol L, Moore L, Sieggreen M. Revised national pressure ulcer advisory panel pressure injury staging system: revised pressure injury staging system. J Wound Ostomy Continence Nurs 2016 Nov/Dec;43(6):585-97. https://doi.org/10.1097/ WON.0000000000000281

2. Wada A, Neto NT, Ferreira MC. Úlceras por pressão. Rev Med 2010 jul/dez;89(3/4): 170-7. https://doi.org/10.11606/ issn.1679-9836.v89i3/4p170-177

3. Agency for healthcare research and quality. Patient Safety Network. Patient Safaty Primer. Never events 2019; [citado 2019 mai 30]. Disponível em: https://psnet.ahrq.gov/ primers/primer/3

4. Borghardt AT, Prado TN, Bicudo SDS, Castro DS, Bringuente MEO. Úlcera por pressão em pacientes críticos: incidência e fatores associados. Rev Bras Enferm 2016 mai/jun;69(3):4607. https://doi.org/10.1590/0034-7167.2016690307i

5. Mervis JS, Phillips TJ. Pressure ulcers: pathophysiology, epidemiology, risk factors, and presentation. J Am Acad Dermatol 2019 Oct;81(4):881-90. https://doi.org/10.1016/j. jaad.2018.12.069

6. Smit I, Harrison L, Letzkus L, Quatrara B. What factors are associated with the development of pressure ulcers in a medical intensive care unit? Dimens Crit Care Nurs 2016 Jan/Feb;35(1):37-41. https://doi.org/10.1097/ DCC.0000000000000153

7. Lima AFC, Castilho V, Baptista CMC, Rogenski NMB, Rogenski KE. Custo direto dos curativos de úlceras por pressão em pacientes hospitalizados. Rev Bras Enferm 2016 mar/abr;69(2):290-7. https://doi.org/10.1590/0034$7167.2016690212 \mathrm{i}$

8. Mendonça PK, Loureiro MDR, Ferreira Júnior MA, Souza AS. Ocorrência e fatores de risco para lesões por pressão em centros de terapia intensiva. Rev enferm UFPE on line 2018 fev;12(2):303-11. https://doi.org/10.5205/1981-8963v12i2a23251p303-311-2018
9. González-Méndez MI, Lima-Serrano M, Martín-Castaño C, Alonso-Araujo I, Lima-Rodríguez JS. Incidence and risk factors associated with the development of pressure ulcers in an intensive care unit. J Clin Nurs 2018 Mar;27(5-6),102837. https://doi.org/10.1111/jocn.14091

10. Raff LA, Waller H, Griffin RL, KerbyJD, Bosarge PL. Identification of risk factors for the development of pressure ulcers despite standard screening methodology and prophylaxis in trauma patients. Adv Skin Wound Care 2016 Jul;29(7):32934. https://doi.org/10.1097/01.ASW.0000484064.86180.18

11. Chen $\mathrm{H}-\mathrm{L}$, Cao $\mathrm{Y}-\mathrm{J}$, Wang J, Huai B-S. Calibration power of the Braden scale in predicting pressure ulcer development. J Wound Care 2016 Nov;25(11):655-9. https://doi. org/10.12968/jowc.2016.25.11.655

12. Gerhardt TE, Silveira DT. Métodos de pesquisa. Universidade Federal do Rio Grande do Sul. Porto Alegre: Editora da UFRGS; 2009; [citado 2020 out 08]. Disponível em: http:// www.ufrgs.br/cursopgdr/downloadsSerie/derad005.pdf

13. Oliveira RA. A pele em diferentes etapas da vida. In: Domansky RC, Borges EL. Manual para prevenção de lesões de pele: recomendações baseadas em evidências. Rio de Janeiro: Editora Rubio; 2012. p. 9-41.

14. Dugaret E, Videau M-N, Faure I, Gabinski C, BourdelMarchasson I, Salles N. Prevalence and incidence rates of pressure ulcers in an emergency department. Int Wound J 2014 Aug;11(4):386-91. https://doi.org/10.1111/j.1742481X.2012.01103.x

15. Jaul E, Calderon-Margalit R. Systemic factors and mortality in elderly patients with pressure ulcers. Int Wound J 2015 Jun;12(3):254-9. https://doi.org/10.1111/iwj.12086

16. Coyer F, Miles S, Gosley S, Fulbrook P, Sketcher-Baker K, Cook $J$-L et al. Pressure injury prevalence in intensive care versus non-intensive care patients: a state-wide comparison. Aust Crit Care 2017 Sep;30(5):244-50. https://doi.org/10.1016/j. aucc.2016.12.003 
17. Black JM, Edsberg LE, Baharestani MM, Langemo D, Goldberg M, McNichol L et al. Pressure ulcers: avoidable or unavoidable? Results of the National Pressure Ulcer Advisory Panel Consensus Conference. Ostomy Wound Manage 2011 Feb; [citado 2020 out 08]; 57(2):24-37. Disponível em: https://pubmed.ncbi.nlm.nih.gov/21350270/

18. Ahn H, Cowan L, Garvan C, Lyon D, Stechmiller J. Risk factors for pressure ulcers including suspected deep tissue injury in nursing home facility residents: analysis of National Minimum Data Set 3.0. Adv Skin Wound Care 2016 Apr;29(4),178-90. https://doi.org/10.1097/01.ASW.0000481115.78879.63

19. Nassaji M, Askari Z, Ghorbani R. Cigarette smoking and risk of pressure ulcer in adult intensive care unit patients. Int J Nurs Pract 2014 Aug;20(4):418-23. https://doi.org/10.1111/ ijn.12141

20. Bly D, Schallom M, Sona C, Klinkenberg D. A model of pressure, oxygenation, and perfusion risk factors for pressure ulcers in the intensive care unit. Am J Crit Care 2016 Mar;25(2):156-64. https://doi.org/10.4037/ajcc2016840
21. Campanili TCGF, Santos VLCG, Strazzieri-Pulido KC, Thomaz PBM, Nogueira PC. Incidência de úlceras por pressão em pacientes de unidade de terapia intensiva cardiopneumológica. Rev Esc Enferm USP 2015 Fev;49(esp):7-14. https://doi.org/10.1590/S0080623420150000700002

22. Campos MGCA, Sousa ATO, organizadores. Feridas complexas e estomias: aspectos preventivos e manejo clínico. João Pessoa: Editora Ideia;2016.

23. Barrois B, Colin D, Allaert F-A. Prevalence, characteristics and risk factors of pressure ulcers in public and private hospitals care units and nursing homes in France. Hosp Pract (1995) 2018 Feb;46(1):30-6. https://doi.org/10.1080/21548331.201 8.1418139

24. Chimentão DMN. Dermatite associada à incontinência. In: Gamba MA, Petri V, Costa MTF. Feridas: prevenção, causas e tratamento. Rio de Janeiro: Santos Editora; 2016. p.174-94. 